\section{The effect of eltrombopag in managing thrombocytopenia associated with tyrosine kinase therapy in patients with chronic myeloid leukemia and myelofibrosis}

\section{Mahran Shoukier, Gautam Borthakur, Elias Jabbour, Farhad Ravandi, Guillermo Garcia-Manero, Tapan Kadia, Jairo Matthews, Lucia Masarova, Kiran Naqvi, Koji Sasaki, Srdan Verstovsek and Jorge Cortes ${ }^{\circ}$}

Department of Leukemia, the University of Texas MD Anderson Cancer Center, Houston, TX, USA

${ }^{\circ}$ Current address: Georgia Cancer Center, Augusta University, Augusta, GA, USA

\section{ABSTRACT}

A pproximately $20-50 \%$ patients with chronic phase chronic myeloid leukemia (CML-CP) treated with tyrosine kinase inhibitors (TKI) or with myelofibrosis (MF) treated with ruxolitinib develop grade $\geq 3$ thrombocytopenia needing treatment interruptions and dose reductions. We conducted a non-randomized, phase II, single-arm study to determine the efficacy of eltrombopag for patients with CML or MF with persistent thrombocytopenia while on TKI or ruxolitinib. Eltrombopag was initiated at $50 \mathrm{mg} /$ day, with dose escalation up to $300 \mathrm{mg}$ daily allowed every 2 weeks. Twenty-one patients were enrolled $(\mathrm{CML}=15, \mathrm{MF}=6)$; with a median age of 60 years (range, 31-97 years). The median platelet count was $44 \times 10^{9} / \mathrm{L}$ (range, $3-49 \times 10^{9} / \mathrm{L}$ ) in CML and $62 \times 10^{9} / \mathrm{L}$ (range, $21-75 \times 10^{9} / \mathrm{L}$ ) in MF. After a median of 18 months (range, 5-77 months), 12 of 15 patients with CML achieved complete platelet response. The median peak platelet count among responders was $154 \times 10^{9} / \mathrm{L}$ (range, $74-893 \times 10^{9} / \mathrm{L}$ ). Among CML patients five could re-escalate the TKI dose and nine improved their response. None of the six patients with MF had a sustained response. Therapy was generally well tolerated. One patient discontinued therapy due to toxicity (elevated transaminases). One patient with CML developed significant thrombocytosis $\left(>1,000 \times 10^{9} / \mathrm{L}\right)$. Another CML patient developed non occlusive deep venous thrombosis in the right upper extremity without thrombocytosis, and one MF patient had myocardial infarction. Eltrombopag may help improve platelet counts in CML patients receiving TKI with recurrent thrombocytopenia. Further studies are warranted (clinicaltrials gov. Identifier: NCT01428635).

\section{Introduction}

Tyrosine kinase inhibitors (TKI) are standard therapy for chronic myeloid leukemia (CML) and myelofibrosis (MF). Five TKI are currently approved for the treatment of CML in various stages, namely imatinib, nilotinib, dasatinib, bosutinib and ponatinib. Although these agents are generally well tolerated, some patients may develop adverse events, with myelosuppression being the most prominent. ${ }^{1-6}$ In most instances myelosuppression is grade 1 or 2 and requires no intervention. However, grade $\geq 3$ thrombocytopenia (platelet $\leq 50 \times 10^{9} / \mathrm{L}$ ) has been reported in $20 \%$ to $50 \%$ of patients. When this occurs, patients are usually managed with treatment interruption until platelets recovery (e.g., above $75 \times 10^{9} / \mathrm{L}$ ) and dose reductions if thrombocytopenia recurs. Ruxolitinib is a JAK2 inhibitor used to manage splenomegaly and diseaseassociated symptoms in patients with MF? The dose limiting toxicity of ruxolitinib was thrombocytopenia ${ }^{8}$ and because of this the two pivotal phase III studies excluded patients with platelets $\leq 100 \times 10^{9} / \mathrm{L}$. Still, thrombocytopenia was reported in $69 \%$ of patients, including $9 \%$ with grade $\geq 3 .{ }^{9}$ In patients with a platelet count of 50 -
Haematologica 2021

Volume 106(11):2853-2858

Correspondence:

JORGE CORTES

jorge.cortes@augusta.edu

Received: May 21, 2020.

Accepted: September 3, 2020.

Pre-published: September 14, 2020.

https://doi.org/10.3324/haematol.2020.260125

(C)2021 Ferrata Storti Foundation

Material published in Haematologica is covered by copyright. All rights are reserved to the Ferrata Storti Foundation. Use of published material is allowed under the following terms and conditions:

https://creativecommons.org/licenses/by-nc/4.0/legalcode. Copies of published material are allowed for personal or internal use. Sharing published material for non-commercial purposes is subject to the following conditions: https://creativecommons.org/licenses/by-nc/4.0/legalcode, sect. 3. Reproducing and sharing published material for commercial purposes is not allowed without permission in writing from the publisher. 
$100 \times 10^{9} / \mathrm{L}$, grade $\geq 3$ thrombocytopenia occurred in $56 \%$ of patients. ${ }^{10}$ Ruxolitinib-associated grade $\geq 3$ thrombocytopenia is also typically managed with dose reductions or interruptions.

Frequent dose interruptions and reductions might decrease TKI efficacy ${ }^{11,12}$ and may still not be sufficient to control thrombocytopenia. Patients who develop myelosuppression have a lower probability of achieving major or complete cytogenetic response (CCyR). ${ }^{11}$ For example, one study reported that patients treated with imatinib who developed grade $\geq 3$ thrombocytopenia had a lower probability of CCyR compared to those who never developed thrombocytopenia ( $35 \%$ vs. $59 \%, P=0.02$, respectively). Similarly, ruxolitinib efficacy is compromised with dose reductions and interruptions. ${ }^{12,13}$ In order to minimize dose reductions and interruptions, hematopoietic growth factors, filgrastim and erythropoietin stimulating agents (erythropoietin and darbepoetin) have been successfully used to manage neutropenia and anemia secondary to TKI in CML, respectively. ${ }^{14,15}$ Interleukin 11 (IL-11) was effective to manage thrombocytopenia associated with TKI in $\mathrm{CML}^{16}$ but use of this agent is associated with significant adverse events including fluid retention and cardiac arrhythmias. Eltrombopag is a non-peptide thrombopoietin receptor agonist that is effective and well tolerated among patients with immune thrombocytopenia, chronic hepatitis C-associated thrombocytopenia and severe aplastic anemia. ${ }^{17-19}$ It has also been safely used in acute myeloid leukemia without evidence of disease progression secondary to eltrombopag. ${ }^{20}$ Here, we report the results from a pilot trial investigating the use of eltrombopag in the management of TKIor ruxolitinib-associated thrombocytopenia among patients with CML and MF.

\section{Methods}

\section{Patients}

We conducted an open-label, non-randomized, phase II study of individualized dosing of eltrombopag. Eligible patients were aged 18 years or older with chronic phase CML receiving treatment with any Food and Drug Administration-approved TKI and experiencing grade $\geq 3$ thrombocytopenia (platelets $\leq 50 \times 10^{9} / \mathrm{L}$ ), or with MF receiving ruxolitinib and with platelets $<100 \times 10^{9} / \mathrm{L}$ (since it is a dose-limiting toxicity and a label threshold for ruxolitinib), in either case after the first 3 months of therapy.

Thrombocytopenia should have been either recurrent (i.e., be at least the second episode of thrombocytopenia) or have necessitated dose reductions of the TKI or ruxolitinib. All patients had signed an informed consent form approved by the Institutional Review Board, and the study was conducted in accordance with the Declaration of Helsinki.

\section{Study design}

Eltrombopag was commenced at $50 \mathrm{mg}$ with dose escalation allowed every 2 weeks to $100 \mathrm{mg}, 150 \mathrm{mg}, 200 \mathrm{mg}$, and $300 \mathrm{mg}$ (a higher dose than per label considering the thrombocytopenia refractoriness on these patients and the intent to continue TKI/ruxolitinib) according to platelet response. For patients of East Asian ancestry, eltrombopag was commenced at $25 \mathrm{mg}$ daily with dose escalation allowed every 2 weeks. The following guideline was used to adjust dosing of eltrombopag: if the platelet count was $>200 \times 10^{9} / \mathrm{L}$ at any time, the daily dose was reduced by $25 \mathrm{mg}$ and re-assessed in 2 weeks; if $>400 \times 10^{9} / \mathrm{L}$, therapy was withheld and platelets assessed twice weekly until platelet count $<150 \times 10^{9} / \mathrm{L}$; therapy could then be resumed with the daily dose reduced by 25 $\mathrm{mg}$. If the platelet count $>400 \times 10^{9} / \mathrm{L}$ after 2 weeks was at the lowest dose, therapy was permanently discontinued. TKI doses were adjusted at the discretion of the treating physician per standard practice. Liver function tests (LFT) (alanine aminotransferase [ALT], aspartate aminotransferase [AST], and bilirubin) were done before the initiation of eltrombopag, every 2 weeks during the dose adjustment phase and following the monthly establishment of a stable dose. When LFT abnormalities were identified, LFT were performed weekly until the abnormalities resolved or stabilized; if ALT/AST levels were $\geq$ three-times the upper limit of normal (ULN): therapy was withheld, we then repeated abnormal liver function tests within 3-5 days; if confirmed abnormal, we monitored LFT weekly until resolved, stabilized, or returned to baseline. If ALT/AST levels $\geq$ three-times the ULN and were progressive, persistent ( $\geq 4$ weeks), accompanied by increased direct bilirubin, or accompanied by clinical signs of liver injury or evidence of hepatic decompensation, eltrombopag was permanently discontinued. Patients who experienced other clinically significant grade 3 or greater toxicity possibly related to eltrombopag, had eltrombopag interruption until toxicity resolved to grade 1 or less. Treatment then was resumed at the immediate lower dose level. Failure to achieve a platelet count $\geq 50 \times 10^{9} / \mathrm{L}$ or $\geq 100 \times 10^{9} / \mathrm{L}$ in CML and MF patients, respectively after 8 weeks of eltrombopag was considered as lack of response.

\section{Statistical analysis}

Simon's optimal two-stage design (Simon, 1989) was used to test the null hypothesis that the proportion of subjects with complete response is $\leq 0.10$ versus the alternative that it is $\geq 0.30$ (i.e., $P o \leq 0.10$ vs. $P a \geq 0.30)$ at alpha $=0.05$ with $80 \%$ power. The design resulted in an expected sample size of 15 and a probability of early termination of 0.736 . The study was designed to study eltrombopag in ten patients in the first stage; the trial would be terminated if one or fewer achieved complete platelet response. Otherwise, the trial would go to the second stage, and 29 patients would be studied. If the total number of patients with complete platelet response were less than or equal to five, the drug would be deemed ineffective.

The MF group was an exploratory group of ten patients to study the safety and activity of eltrombopag in patients with MF treated with ruxolitinib. We considered the activity promising if three or more patients out of ten achieved complete platelet response. For safety monitoring in the cohort with MF, accrual would stop if, at any time, four of ten patients encounter grade 3 or more nonhematological toxicity or progression to acute leukemia. As an additional safety procedure, we observed the first three MF patients on trial for at least 3 months before other patients were accrued.

\section{Response definitions}

Complete platelet response was defined as platelet count $\geq 50 \times 10^{9} / \mathrm{L}$ for $\mathrm{CML}$, and $\geq 100 \times 10^{9} / \mathrm{L}$ for MF that was sustained for $\geq 3$ months while continuing TKI or ruxolitinib therapy or with sustained ( $\geq 3$ months) re-escalation of TKI dose without recurrence of thrombocytopenia. Criteria for CML and MF response were previously defined. ${ }^{21,22}$ The target response was a complete response in at least $30 \%$ of patients.

\section{Results}

Twenty-one patients were enrolled: 15 with CML and six with MF. Their median age was 60 years (range, 31-97) and their clinical characteristics are shown in Table 1. Median 
duration of disease was 2.2 years (range, 0.5-29 years) for patients with CML and 2 years (range, 0.3-3.6 years) for patients with MF. At the time of enrollment, patients with CML were receiving the following TKI: dasatinib $(n=5)$, ponatinib $(n=4)$, nilotinib $(n=3)$, bosutinib $(n=2)$, and imatinib $(n=1), 27 \%$ were receiving their first TKI, $27 \%$ the second TKI, $27 \%$ the third, and $19 \%$ the fourth or later TKI. The median platelet count was $44 \times 10^{9} / \mathrm{L}$ (range, $3-49 \times 10^{9} / \mathrm{L}$ ) in patients with CML and $62 \times 10^{9} / \mathrm{L}$ (range, $21-75 \times 10^{9} / \mathrm{L}$ ) in those with MF. Cytogenetic response for patients with CML at baseline were partial in three, minor in six, and none in six. Prior therapies in MF patients were an investigational JAK2 inhibitor, and interferon $\alpha-2$ in one patient each. The median dose of ruxolitinib was $10 \mathrm{mg}$ (range, 10$30 \mathrm{mg}$ ) (Table 1). Eltrombopag dose distribution is summarized in Table 2 .

After a median duration of treatment of 18 months (range, 5-77 months), 12 of the 15 (80\%) patients with CML achieved a complete platelet response with doses of eltrombopag of 50-300 mg per day. The median peak platelet count among responders was $154 \times 10^{9} / \mathrm{L}$ (range, 74$893 \times 10^{9} / \mathrm{L}$ ). The median time to best response was 6 months (range, 2.1-13 months). Ten patients had sustained platelet recovery after stopping eltrombopag. The median duration for sustained platelet response was 45 months (range, 3-69 months). The three patients who did not achieve a complete platelet response had only minor changes in platelet count while they were taking eltrombopag (from $3 \times 10^{9} / \mathrm{L}$ to $8 \times 10^{9} / \mathrm{L}, 19 \times 10^{9} / \mathrm{L}$ to $45 \times 10^{9} / \mathrm{L}$, and from $42 \times 10^{9} / \mathrm{L}$ to $46 \times 10^{9} / \mathrm{L}$, respectively).

Two patients (one each of CML and MF) had improvement in hemoglobin of over $2 \mathrm{~g} / \mathrm{dL}$ from baseline (from 8.2 $\mathrm{g} / \mathrm{dL}$ to $10.6 \mathrm{~g} / \mathrm{dL}$, and from $9.4 \mathrm{~g} / \mathrm{dL}$ to $11.4 \mathrm{~g} / \mathrm{dL}$, respectively), Hemoglobin improvement was sustained over 21.5 and 2 months respectively while patients were taking eltrombopag. Hemoglobin levels declined after interruption of eltrombopag. One patient with CML had an absolute neutrophil count recovery to $>1 \times 10^{9} / \mathrm{L}$ (baseline neutrophils $0.71 \times 10^{9} / \mathrm{L}$ ). Absolute neutrophil count improvement was sustained for $>6$ months while on eltrombopag. Absolute neutrophil count then declined after interruption of eltrombopag. The TKI doses and duration for patients with CML post enrollment are summarized in Table 1.

Nine patients with CML experienced an improvement in the cytogenetic response during the observation period (all of them had sustained platelet recovery after stopping eltrombopag); one from none to complete, two from minor to complete, four from minor to partial, and two from partial to complete (Table 3 ). In five patients with CML the TKI dose was increased and maintained while continuing eltrombopag. Dasatinib daily dose was increased from 50 $\mathrm{mg}$ to $100 \mathrm{mg}$ in three patients, nilotinib dose was increased in one patient form $150 \mathrm{mg}$ twice daily to $200 \mathrm{mg}$ twice daily, and one patient had an increase in ponatinib dose from $15 \mathrm{mg}$ every other day to $15 \mathrm{mg}$ daily. There were no TKI dose-limiting toxicities in patients who increased their TKI doses. The dose increase was associated with improvement in CML response in four of these five patients. In the five CML patients who had a cytogenetic response but did not have TKI dose escalation, the improvement in cytogenetic response was noticed while patients were on eltrombopag. Three CML patients had a switch in their TKI (Online Supplementary Table S1). All three of these patients had already some improvement in thrombocytopenia before switching their TKI, with the change indicated for
Table 1. Baseline characteristics.

\begin{tabular}{|c|c|c|}
\hline Characteristics & $N(\%)$ & Median [range] \\
\hline Age, years & & $60[31-97]$ \\
\hline Male & $12(58)$ & \\
\hline \multicolumn{3}{|l|}{ Disease } \\
\hline CML & $15(71)$ & \\
\hline $\mathrm{MF}$ & $6(29)$ & \\
\hline \multicolumn{3}{|c|}{ Stage of CML at start of eltrombopag } \\
\hline Chronic phase & $15(100)$ & \\
\hline \multicolumn{3}{|c|}{ Disease duration prior to Eltrombopag initiation (years) } \\
\hline CML & & $2.2[0.5-29]$ \\
\hline $\mathrm{MF}$ & & $2[0.3-3.6]$ \\
\hline Hemoglobin (all patients), g/dL & & $11.6[8.2-13.4]$ \\
\hline WBC (all patients), x109/L & & $4.6[1-71.3]$ \\
\hline Platelet count (CML), x10 $/ \mathrm{L}$ & & $44[3-49]$ \\
\hline Platelet count (MF), x109/L & & $62[21-75]$ \\
\hline TKI (CML patients) & dose $\mathrm{mg} / \mathrm{d}$ & \\
\hline Dasatinib & $5(33)$ & $50[50-100]$ \\
\hline Ponatinib & $4(27)$ & $11.25[7.5-30]$ \\
\hline Nilotinib & $3(20)$ & $300[150-600]$ \\
\hline Bosutinib & $2(13)$ & 300 \\
\hline Imatinib & $1(7)$ & 400 \\
\hline Ruxolitinib (MF patients) & $6(100)$ & $10[10-30]$ \\
\hline \multicolumn{2}{|c|}{ Time on TKI before eltrombopag, years } & $2.1[0.5-14]$ \\
\hline Time on ruxolitinib before eltror & , months & $3[3-18]$ \\
\hline \multicolumn{3}{|c|}{ Cytogenetic response prior to eltrombopag (CML) } \\
\hline None & $6(40)$ & \\
\hline Minor & $6(40)$ & \\
\hline Partial & $3(20)$ & \\
\hline
\end{tabular}

CML: chronic myeloid leukemia; MF: myelofibrosis; TKI: tyrosine kinase inhibitor.TKI was on hold in six patients with CML at time of enrollment because of grade 3 thrombocytopenia; WBC: white blood cells.

Table 2. Eltrombopag dose distribution, mg per day (all patients).

\begin{tabular}{lcc} 
& \multicolumn{1}{c}{$N(\%)$} & \\
Dose & Maximum dose & Dose at last follow-up \\
0 & 0 & $2(10)$ \\
25 & $1(5)$ & 0 \\
\hline 50 & $3(14)$ & $3(14)$ \\
100 & 0 & $3(14)$ \\
150 & $2(10)$ & $2(10)$ \\
200 & $2(10)$ & $1(5)$ \\
275 & 0 & $1(5)$ \\
300 & $13(61)$ & $9(42)$ \\
\hline
\end{tabular}

other non-hematologic adverse events in one patient and the inefficacy of the TKI in the other two patients. None of the six patients with MF responded (i.e., none had a sustained increase in platelet count to $\geq 100 \times 10^{9} / \mathrm{L}$ ); minor upward transient variations in platelet counts were seen in three patients (from $21 \times 10^{9} / \mathrm{L}$ to $28 \times 10^{9} / \mathrm{L}, 41 \times 10^{9} / \mathrm{L}$ to $55 \times 10^{9} / \mathrm{L}$ and from $65 \times 10^{9} \mathrm{~L}$ to $75 \times 10^{9} / \mathrm{L}$, respectively).

As of the date of this report, 20 patients were off study because of a lack of response $(n=9)$, stem cell transplant $(n=2)$, death $(n=2)$, patient's wish $(n=1)$, adverse events $(n=2)$, TKI discontinuation $(n=1)$, loss to follow-up $(n=1)$ 


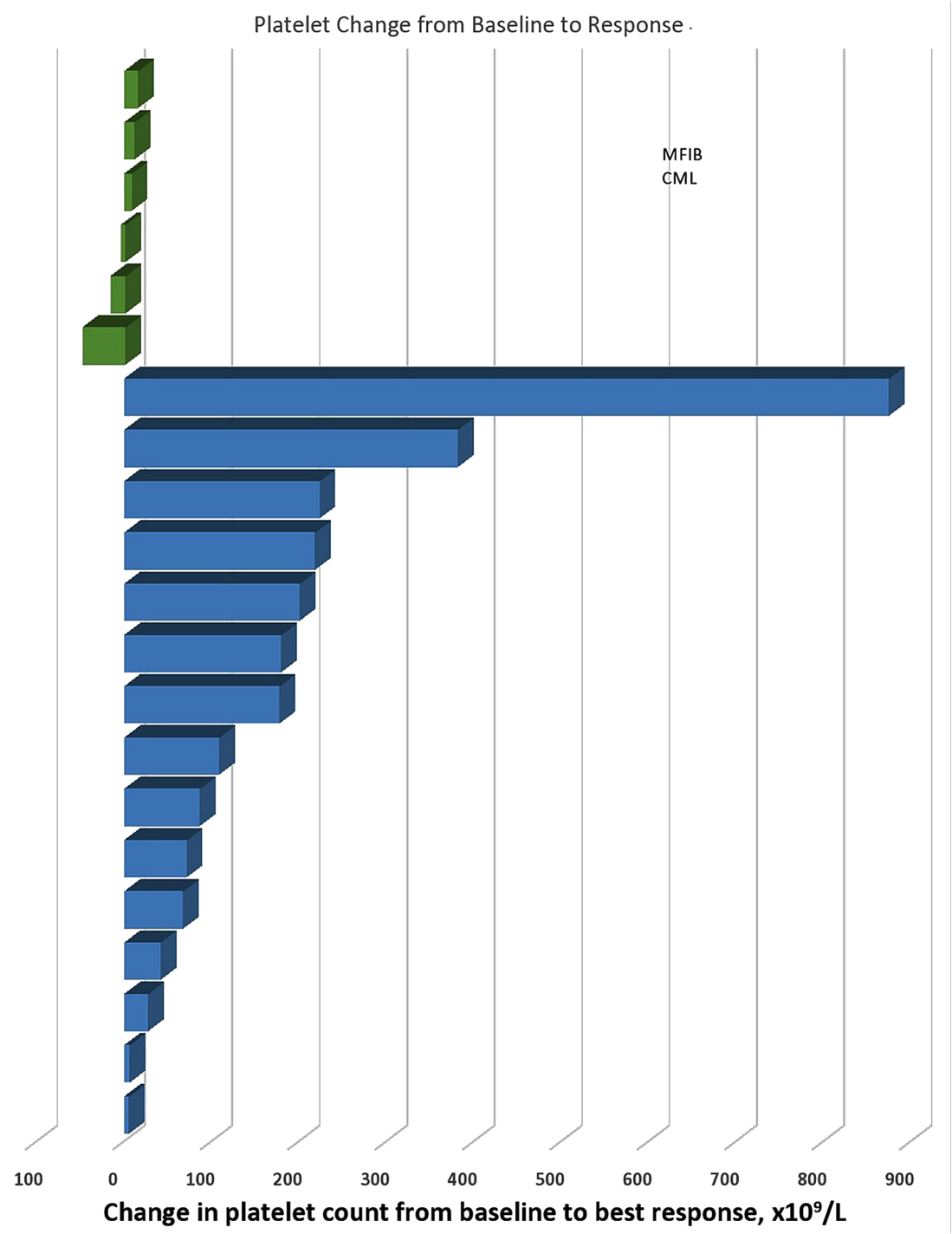

Figure 1. Platelet change from baseline to response. *Each blue bar reflects change in platelet count in a chronic myeloid leukemia patient, while each green bar reflects change in platelet count in a myelofibrosis patient.

and stable platelets $(n=2)$. The two deaths on study were not related to treatment. One death was secondary to infectious complication in a patient with MF. The second death was secondary to hemorrhagic shock in a CML patient. This patient, treated with dasatinib, developed hepatosplenomegaly and ascites while on study but the etiology was not known. There was no evidence of portal vein thrombosis on CT abdomen/pelvis. Both eltrombopag and dasatinib were held and she had no platelets response. The platelet count was $10 \times 10^{9} / \mathrm{L}$ at the time of death due to severe gastrointestinal and genitourinary bleeding.

Therapy was well tolerated in most patients, but two patients on ponatinib developed thrombotic events. Two months after eltrombopag discontinuation due to termination of the study, one patient with CML developed significant thrombocytosis $\left(>1,000 \times 10^{9} / \mathrm{L}\right)$ with a white blood cell count of $9.5 \times 10^{9} / \mathrm{L}, 3 \%$ basophils, and $2 \%$ peripheral blast accompanied by headache and eye pain. Ophthalmoscopic examination was suggestive of bilateral plaques or thrombosis in the retinal vasculature but fluoroscopic evaluation did not reveal retinal vasculature blockage. Ponatinib was discontinued and thrombocytosis was managed with hydroxyurea. The aforementioned symptoms resolved. There was no cytogenetic response prior or after starting eltrombopag. Seven months after stopping eltrombopag, the patient had a persistent increase in blasts to $13 \%$ without a lack of hematologic response and she was then started on a clinical trial with an investigational TKI. Another CML patient developed non-occlusive deep venous thrombosis in the right upper extremity without thrombocytosis while on ponatinib 4 months after the study was terminated. One MF patient who had a history of coronary artery disease status post coronary artery bypass surgery developed myocardial infarction (MI) while on eltrombopag. This patent had then worsening increase in bone marrow fibrosis from grade 2 to grade 3 and was taken off study 40 days after MI. No further additional thrombotic/thromboembolic complications in CML and MF patients observed during or after the study (Online Supplementary Table S2).

One patient (CML) discontinued therapy due to toxicity (elevation of liver function tests). Grade 3/4 toxicities irrespective of attribution listed in Table 4. One patient with MF had an increase in bone marrow fibrosis from grade 2 to grade 3 . That patient had an increase in blast from $3 \%$ to $8 \%$ in the peripheral blood and an increase from $1 \%$ to $6 \%$ in the bone marrow while he was on study but with an improvement in hemoglobin. There was no change in the patient's disease other than this change in blast percentage. The patient was taken off study for lack of platelet response and later started on another clinical trial (PRM-151 + ruxoli- 
tinib). No progression of disease has been documented in any other patients. No clonal evolution was observed in patients with prolonged eltrombopag use.

\section{Discussion}

Thrombocytopenia is a common adverse event in patients with CML and MF who are treated with TKI and ruxolitinib, respectively. ${ }^{10,23}$ In most instances, thrombocytopenia is transient, occurs early during treatment initiation, and can be successfully managed with transient treatment interruptions and occasionally dose adjustments. However, in some patients thrombocytopenia can be persistent and more severe requiring frequent treatment interruptions and dose reductions, which might adversely influence treatment outcome. ${ }^{11}$ To that end, rIL-11 was successfully used in CML patients for the management of TKI associated thrombocytopenia. ${ }^{16}$ The main limitation of use of rIL-11 in the management of chemotherapy-induced thrombocytopenia in solid malignancies was the narrow therapeutic window with significant fluid retention and occasional arrhythmias. However, at lower doses used in CML, it was well tolerated ${ }^{24,25}$ with grade 1 or 2 peripheral edema observed in six patients (43\%).

Eltrombopag is a second generation oral thrombopoietin receptor agonist that has induced improvement of thrombocytopenia in patients with immune-mediate thrombocytopenia (ITP) or aplastic anemia. The EXTEND trial demonstrated that long-term use of eltrombopag was effective in maintaining for more than 6 months platelet counts of $50 \times 10^{9} / \mathrm{L}$ or more and reducing bleeding in most patients with ITP. Addition of eltrombopag to immunosuppressive treatment also markedly increased overall and complete hematologic response rates in treatment-naive severe aplastic anemia. ${ }^{26}$ Here we describe the use of eltrombopag in the management of TKI-related thrombocytopenia in CML and MF. Our results suggest clinical benefit in most patients with CML with a generally favorable safety profile, although two patents (both on ponartinib) had thrombotic events. In contrast, no response was observed in patients with MF.

Theoretical concerns about the use of eltrombopag in this setting include increase in marrow blasts and possible transformation to advanced phases, thrombotic events including portal vein thrombosis, and increase in marrow fibrosis. We did not observe any instance of transformation in our series, in concordance with pre-clinical and clinical data showing no evidence of worsening leukemia. ${ }^{20,27}$ There was also no increase in marrow fibrosis in CML patients. Our series is small so the lack of such events should be considered as preliminary but reassuring. The most common adverse event was LFT elevation, but these were generally transient, reversible and manageable with dose adjustments. However, in one case it led to discontinuation of eltrombopag because of recurrent transaminitis. Two patients who received ponatinib $(50 \%)$ had thrombotic events while on eltrombopag, this might raise the precaution of using ponatinib in conjunction with eltrombopag in CML patients.

Despite the median disease duration of 2.2 years and multiple TKI use in CML patients before enrollment, eltrombopag demonstrated clinical efficacy with complete platelet response of $80 \%$ (12 of 15). This compares favorably to what was reported with rIL-11. ${ }^{16}$ More important,
Table 3. Response to eltrombopag in chronic myeloid leukemia patients.

\begin{tabular}{lcc} 
Before eltrombopag & $\begin{array}{c}\text { Cytogenetic response (CML patients) } \\
\text { On eltrombopag }\end{array}$ & No. \\
None & Complete & 1 \\
\hline Minor & Complete & 2 \\
Minor & Partial & 4 \\
\hline Partial & Complete & 2 \\
Before eltrombopag & On eltrombopag & P \\
55 & 73.5 & 0.3 \\
\hline
\end{tabular}

CML: chronic myeloid leukemia;TKI: tyrosine kinase inhibitor.

Table 4. Treatment emergent adverse events.

\begin{tabular}{|c|c|c|}
\hline \multirow[t]{2}{*}{ Event } & \multicolumn{2}{|c|}{ No. (\%) } \\
\hline & Any & Grade 3-4 \\
\hline Elevated AST/ALT & $9(16)$ & $5(22)$ \\
\hline Fatigue & $7(12)$ & $2(8)$ \\
\hline Infection & $7(12)$ & $6(25)$ \\
\hline Diarrhea & $4(7)$ & $1(5)$ \\
\hline Rash & $3(5)$ & - \\
\hline Insomnia & $3(5)$ & - \\
\hline Hyperglycemia & $2(4)$ & $2(8)$ \\
\hline HTN & $2(4)$ & $2(8)$ \\
\hline HLD & $2(4)$ & $2(8)$ \\
\hline Pleural effusion & $2(4)$ & $1(4)$ \\
\hline Headache & $2(4)$ & - \\
\hline Hyperbilirubinemia & $2(4)$ & - \\
\hline Limb edema & $2(4)$ & - \\
\hline Blurred vision & $2(4)$ & - \\
\hline Peripheral neuropathy & $2(4)$ & - \\
\hline Thrombocytosis & $1(2)$ & $1(4)$ \\
\hline Chest pain & $1(2)$ & $1(4)$ \\
\hline Myocardial infarction & $1(2)$ & $1(4)$ \\
\hline Periorbital edema & $1(2)$ & - \\
\hline Non occlusive deep venous thrombosis & $1(2)$ & \\
\hline
\end{tabular}

ALT: alanine aminotransferase; AST: aspartate aminotransferase; HTN: hypertension; HLD: hyperlipidemia.

nine patients (60\%) had improvement in cytogenetic responses, likely the result of a more sustained therapy with TKI. Notably, as doses of eltrombopag were increased, LFT elevations were noted in some patients. Conversely, eltrombopag dose interruptions or reductions due to such events or to platelets reaching $>200 \times 10^{9} / \mathrm{L}$, occasionally resulted in a drop-in platelet counts. Thus, close monitoring and dynamic management is required, at least during the initial stages of therapy to obtain the maximum effect while maintaining safety. The lack of efficacy among patients with MF could be in part secondary to advanced disease, or possible antagonism between the two medications. Thrombopoietin agonist are dependent on JAK-stat pathway which is inhibited by ruxolitinib. ${ }^{28}$

Our study has several limitations. It was a small study, and it did not accrue to the target sample size of 29 patients due to slow enrollment making the observation preliminary and requiring confirmation. We also do not have evidence or investigation of any immune mechanisms associated 
with thrombocytopenia, although we believe it is unlikely that these patients with CML had an immune mediated thrombocytopenia, and uncommon occurrence in this setting.

In conclusion, our findings show that eltrombopag doses up to $300 \mathrm{mg}$ may alleviate TKI-associated thrombocytopenia in some patients with CML. No similar benefit has been observed in patients with MF treated with ruxolitinib. Although generally safe, thrombotic events were noted that deserve further investigation, particularly when used in combination with ponatinib. Additional studies are warranted to confirm these observations.

\section{Disclosures}

$M S, G G-M, J M, L M, K N, K S$, and $S V$ have no conflicts of interset to disclose; GB sit on the advisory board of Novartis; EJ has received consultancy honoraria from BMS, Novartis, Pfizer, and
Ariad; FR has received honoraria and is a member of the advisory board of Novarts; TK has received honoraria from Novartis; JC has received research support from BMS, Novartis, Ariad, Chemgenex, and Pfizer.

\section{Contributions}

MS analyzed the data, and wrote the paper; GB designed, and performed research; IM performed research, and analyzed the data; JC designed, performed research, analyzed the data, and wrote the paper; and all authors contributed to data collection, reviewed and approved the manuscript, and shared final responsibility for the decision to submit.

\section{Funding}

The study was supported in part by MD Anderson Cancer Center Support Grant CA016672 (PI: Dr. Ronald DePinho) and Novartis.

\section{References}

1. Kantariian HM, Cortes JE, O'Brien S, et al. Imatinib mesylate therapy in newly diagnosed patients with Philadelphia chromosome-positive chronic myelogenous leukemia: high incidence of early complete and major cytogenetic responses. Blood. 2003;101(1):97-100

2. Kantarjian H, Shah NP, Hochhaus A, et al. Dasatinib versus imatinib in newly diagnosed chronic-phase chronic myeloid leukemia. N Engl J Med. 2010; 362(24): 2260-2270.

3. Cortes JE, Jones D, O'Brien S, et al. Nilotinib as front-line treatment for patients with chronic myeloid leukemia in early chronic phase. J Clin Oncol. 2010; 28(3):392-397.

4. Cortes JE, Kim DW, Kantariian HM, et al. Bosutinib versus imatinib in newly diagnosed chronic-phase chronic myeloid leukemia: results from the BELA trial. J Clin Oncol. 2012;30(28):3486-3492.

5. Cortes JE, Kim DW, Pinilla-Ibarz J, et al. A phase 2 trial of ponatinib in Philadelphia chromosome-positive leukemias. N Engl J Med. 2013;369(19):1783-1796.

6. Hochhaus A, Kantariian HM, Baccarani M, et al. Dasatinib induces notable hematologic and cytogenetic responses in chronicphase chronic myeloid leukemia after failure of imatinib therapy. Blood. 2007; 109(6):2303-2309.

7. Incyte Corporation (2016) Jakafi (ruxolitinib) [package insert].https://www.jakafi. com/ pdf/prescribing-information.pdf. Accessed 1 March 2016.

8. Verstovsek S, Kantariian H, Mesa RA, et al. Safety and efficacy of INCB018424, a JAK1 and JAK2 inhibitor, in myelofibrosis. N Engl J Med. 2010;363(12):1117-1127.

9. Harrison C, Kiladjian JJ, Al-Ali HK, et al. JAK inhibition with ruxolitinib versus best available therapy for myelofibrosis. $\mathrm{N}$ Engl J Med. 2012;366(9):787-798.

10. Talpaz M, Paquette R, Afrin L, et al. Interim analysis of safety and efficacy of ruxolitinib in patients with myelofibrosis and low platelet counts. J Hematol Oncol. 2013; 6(1):81.

11. Sneed TB, Kantarjian HM, Talpaz M, et al. The significance of myelosuppression dur- ing therapy with imatinib mesylate in patients with chronic myelogenous leukemia in chronic phase. Cancer. 2004; 100(1):116-121.

12. Verstovsek S, Gotlib J, Gupta V, et al. Management of cytopenias in patients with myelofibrosis treated with ruxolitinib and effect of dose modifications on efficacy outcomes. Onco Targets Ther. 2013;7:1321.

13. Verstovsek S, Mesa RA, Gotlib J, et al. A double-blind, placebo-controlled trial of ruxolitinib for myelofibrosis. N Engl J Med. 2012;366(9):799-807.

14. Quintas-Cardama A, Kantariian H, O'Brien $S$, et al. Granulocyte-colony-stimulating factor (filgrastim) may overcome imatinibinduced neutropenia in patients with chronic-phase chronic myelogenous leukemia. Cancer. 2004;100(12):2592-2597.

15. Cortes J, O'Brien S, Quintas A, et al. Erythropoietin is effective in improving the anemia induced by imatinib mesylate therapy in patients with chronic myeloid leukemia in chronic phase. Cancer. 2004:100(11):2396-2402

16. Aribi A, Kantarjian H, Koller C, et al. The effect of interleukin 11 on thrombocytopenia associated with tyrosine kinase inhibitor therapy in patients with chronic myeloid leukemia. Cancer. 2008; 113(6):1338-1343.

17. Saleh MN, Bussel JB, Cheng G, et al. Safety and efficacy of eltrombopag for treatment of chronic immune thrombocytopenia: results of the long-term, open-label EXTEND study. Blood. 2013;121(3):537545

18. McHutchison JG, Dusheiko G, Shiffman ML, et al. Eltrombopag for thrombocytopenia in patients with cirrhosis associated with hepatitis C. N Engl J Med. 2007; 357(22):2227-2236.

19. Townsley DM, Dumitriu B, Sheinberg P, Desmond P. Eltombopag added to standard immunosuppressin for aplastic anemia accelerates count recovery and increases response rates. Blood. 2015, 126(23)LBA-2LBA-2.

20. Platzbecker U, Wong RS, Verma A, et al. Safety and tolerability of eltrombopag versus placebo for treatment of thrombocytopenia in patients with advanced myelodysplastic syndromes or acute myeloid leukaemia: a multicentre, randomised, placebo-controlled, double-blind, phase 1/2 trial. Lancet Haematol. 2015; 2(10):e417-426.

21. Baccarani M, Cortes J, Pane F, et al. Chronic myeloid leukemia: an update of concepts and management recommendations of European LeukemiaNet. J Clin Oncol. 2009; 27(35):6041-6051.

22. Tefferi A, Barosi G, Mesa RA, et al. International Working Group (IWG) consensus criteria for treatment response in myelofibrosis with myeloid metaplasia, for the IWG for Myelofibrosis Research and Treatment (IWG-MRT). Blood. 2006; 108(5):1497-1503

23. Jain P, Kantarjian H, Alattar ML, et al. Longterm molecular and cytogenetic response and survival outcomes with imatinib 400 $\mathrm{mg}$, imatinib $800 \mathrm{mg}$, dasatinib, and nilotinib in patients with chronic-phase chronic myeloid leukaemia: retrospective analysis of patient data from five clinical trials. Lancet Haematol. 2015;2(3):e118-128.

24. Bhatia M, Davenport V, Cairo MS. The role of interleukin-11 to prevent chemotherapy-induced thrombocytopenia in patients with solid tumors, lymphoma, acute myeloid leukemia and bone marrow failure syndromes. Leuk Lymphoma. 2007:48(1):9-15

25. Cantor SB, Elting LS, Hudson DV, Rubenstein EB. Pharmacoeconomic analysis of oprelvekin (recombinant human interleukin-11) for secondary prophylaxis of thrombocytopenia in solid tumor patients receiving chemotherapy. Cancer. 2003;97(12):3099-3106.

26. Townsley DM, Scheinberg P, Winkler T, et al. Eltrombopag added to standard immunosuppression for aplastic anemia. $\mathrm{N}$ Engl J Med. 2017;376(16):1540-1550.

27. Roth M, Will B, Simkin G, et al Eltrombopag inhibits the proliferation of leukemia cells via reduction of intracellular iron and induction of differentiation. Blood. 2012;120(2):386-394.

28. Erickson-Miller CL, Delorme E, Tian SS, et al. Preclinical activity of eltrombopag (SB497115), an oral, nonpeptide thrombopoietin receptor agonist. Stem Cells. 2009; 27(2):424-430. 